\title{
Cluster Based Hierarchical Routing Protocol for WSN with Energy Efficiency
}

\author{
L. Malathi and R. K. Gnanamurthy
}

\begin{abstract}
Recent advances in the field of electronics and communication leads to development of tiny battery enabled sensor nodes. These sensor nodes are randomly deployed as a wireless sensor network for sensing environment. Over the years, the most important problem in wireless sensor network is to develop a routing protocol that maximize the life time of network. Each node in a sensor network becomes useless after wasting its energy completely because its power totally depends on the embedded battery. So we should employ the routing protocols which can consume the energy of nodes efficiently. Many protocols for energy efficient routing in sensor networks have been suggested but LEACH and PEGASIS are most well-known protocols based on Clustering. However LEACH consumes energy heavily in the head nodes and the head nodes tend to die early and PEGASIS - which is known as a better energy efficient protocol has a long transfer time from a source node to sink node. We proposed a new three stage routing protocol which combines LEACH and PEGASIS. And we introduced the concept called Cluster Head Set which is responsible for transferring the data. The Headed selection is similar to LEACH. In addition to Header Head set members are selected. The Data forwarding technique is similar to PEGASIS. The Simulation Results shows that this protocol consumes the energy of the node efficiently and improves the life time of the Network and also it reduces the redundant transmission of the data. For set of 100 Nodes the proposed algorithm gives 50 percent better performance than LEACH and 6 percent better performance than PAGASIS in terms of network life time and also it gives 61 percent better performance in terms of redundant transmission.
\end{abstract}

Index Terms-Clustering, cluster head set, leach, PEGASIS, routing, sensor network, life time.

\section{INTRODUCTION}

Due to the recent technology advances, the manufacturing of small and low-cost sensors has become technically and economically feasible. Continued advances of wireless communication technologies have enabled the deployment of large scale wireless sensor networks (WSNs) [1]. Each sensor node is equipped with a limited battery-supplied energy which makes energy consumption a critical issue. Most of the time, the sensor nodes spent its energy in forwarding the data. Innovative techniques are highly required to improve the routing algorithm and thereby increase energy efficiency and prolong the lifetime of WSNs.

Distinguished from traditional wireless communication

Manuscript received January 2, 2014; revised May 30, 2014

L. Malathi is with the Computer Science and Engineering Department, Vivekanandha College of Engineering for Women, Thiruchengode, Tamil Nadu, India (e-mail: malasumathi@gmail.com).

R. K. Gnanamurthy is with the ECE, SKP Engineering College, Thiruvannamalai, Tamil Nadu, India. networks, for example, cellular systems and mobile ad hoc networks, WSNs have unique characteristics such as denser level of node deployment, higher unreliability of sensor nodes, and severe energy, computation, and storage constraints [2], which present many new challenges in the development and application of WSNs. In wireless sensor network, the energy is mainly consumed by three processes: data transmission, signal processing and hardware operation. It is proved that $70 \%$ of energy consumption is caused during data transmission process [3]. Therefore, the process of data transmission should be optimized in order to maximize the network lifetime.

There have been many sensor network routing protocols proposed to solve various kinds of problems, demanded in sensor networks. Presently, widely suggested routing techniques are distributed among several classes which are: flat, hierarchical, and location-based techniques. Many energy-efficient solutions have been put out. An approach that is likely to succeed is the use of a hierarchical structure [4]. Normally considered, the most important part in sensor networks is the life span of the nodes. Each node in a sensor network becomes useless after wasting its energy completely because its power totally depends on the embedded battery and it is unlikely to be returned due to the remoteness of the area. LEACH and PEGASIS are the two energy efficient routing protocols devised to extend the life span of the nodes in the networks. In sensor networks, once a node starts to die then the whole network is considered to be dead since the first node triggers others nodes to die soon as well.

LEACH (Low Energy Adaptive Clustering Hierarchy) is a widely accepted hierarchical routing protocol, and PEGASIS (Power-Efficient GAthering in Sensor Information Systems), which is devised to make up for the weak points in LEACH, is notable as well. However, the drawbacks of LEACH (or LEACH-C) lie in the fact that the headers become exhausted earlier than other nodes, and PEGASIS, known as more efficient than LEACH, also has a weak point to make the route from the source node to the sink node significantly lengthy. The Proposed Routing Protocol is the combined approach of LEACH and PEGASIS with reducing energy consumption and prolongs the life time of the network.

\section{RELATED WORKS}

Various methods for minimizing energy consumption in wireless sensor network have been proposed such as by Heinemann et al. [5] who described the LEACH protocol as a hierarchical self-organized cluster based approach for monitoring application. The data collection area of the data is randomly divided into clusters. LEACH uses time 
division multiple access (TDMA), to transmit data from the sensor nodes to the cluster head. Then $\mathrm{CH}$ aggregates the data and transmits it to the base station for processing. One of the features of LEACH is localized coordination and control for the formation and operation of clusters. The cluster head rotate randomly.

In [6] Lindsey et al. came about the proposition of PEGASIS which is an extension of LEACH. It eliminates the overhead of dynamic cluster formation created by LEACH. In this protocol, the nodes transmit to the $\mathrm{CH}$ and transmission of data is done by the cluster head, which is selected in a rotational manner, to the BS. PEGASIS protocol is found to save more energy and is more robust in node failure when compared to LEACH. In HEED, author introduces a variable known as cluster radius which defines the transmission power to be used for intra-cluster broadcast [7]. HEED terminates within a constant number of iterations, and achieves fairly uniform distribution of cluster heads across the network. The authors in [8] determine the optimal cluster size in network for analyzing the problem of prolonging network lifetime. The authors in [9] maximize the lifetime of network by using dynamic clusters. In [10] the authors optimize cluster size to reduce the converge hole problem.

\section{THE PROPOSED APPROACH}

In this paper a cluster based hierarchical routing protocol for wireless sensor network which uses optimum energy is proposed, which is a three layer protocol where a number of clusters cover the whole region. After cluster formation the cluster, the four nodes are selected as head set members based on the remaining energy of the sensor nodes. A cluster head set member with maximum energy is selected as Active Cluster Head. All the active cluster head are connected to form the data forwarding path to the Base station. Proposed protocol introduces a concept of cluster head-set (CHS) instead of a cluster head. At one time, only one member of set is active and the remaining is in sleep mode.

\section{THE PROPOSED ARCHITECTURE}

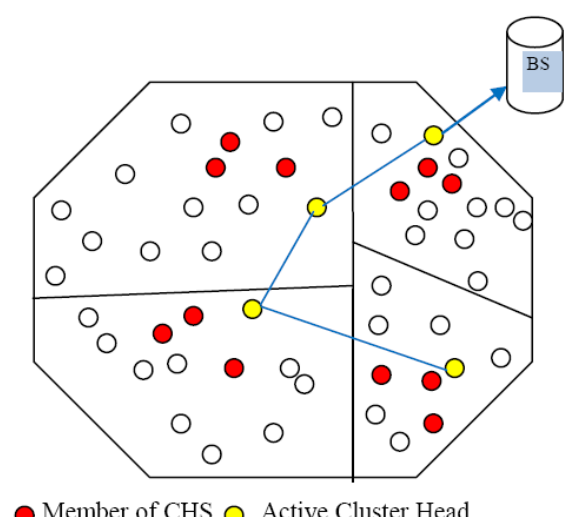

Chain Path

Fig. 1. The proposed algorithm.

In Fig. 1, the yellow spots are the Active cluster headers, red spots are the members of cluster head set and the black spots are their nodes.

\section{A. Cluster Head Set Selection}

The new protocol organizes clusters with the headers chooses by the BS as LEACH does. In addition to the Header Selection, These cluster heads send a short range advertisement broadcast message. The sensor nodes receive the advertisements and choose their cluster heads based on the signal strength of the advertisement messages. Each sensor node sends an acknowledgment message to its cluster head. The cluster heads choose a set of associate heads based on the signal strength of the acknowledgments. A cluster head-set consists of a cluster head and the associates. The Cluster head-set member is responsible to send messages to the base station. After cluster formation, $\mathrm{CHs}$ are designated which act as a leader in each clusters. Cluster heads are saddled with the responsibility for data aggregation and performing routing for its cluster member's information to the base station. Also, the clusters that consist of many nodes have a higher burden than clusters with fewer nodes as the $\mathrm{CHs}$ for those large-sized clusters have to receive, aggregate and transmit more data.

\section{B. Chain Formation}

We apply the Greedy algorithm used in PEGASIS to chain the headers. All member nodes in each cluster transmits its data to the header, and all the headers send the data to its leader node along the chain, finally the leader node transfers the collected data to the BS. The leader node is not statically selected but is dynamically decided in the order of the remaining amount of energy of the all active cluster head to avoid one certain node to die earlier than others.

\section{Path Management}

The Proposed approach uses the energy threshold Eth to improve the header selection method of LEACH and to use energy more efficiently. The Energy level of the active cluster head is monitored if it is below the threshold value then this cluster head goes to sleep mode, another member of cluster head set is elected as new active cluster head.

Energy values of cluster head set nodes are calculated using the following formula

$$
E=E_{\text {rem }} / E_{\text {init }} * C_{p}
$$

In above formula, $E_{\text {rem }}$ indicates the remaining amount of energy for the node, $E_{\text {init }}$ is the amount of its initial energy and $C_{p}$ indicates the proportion of the number of the headers to the number of all nodes in the network. In this paper, we uses $5 \%$ for the proportion, which is the same ratio that $\mathrm{LEACH}$ uses. When a newly-calculated $E$ is less than the value of the $E$ th, Then the node with the Maximum $E$ from the head set becomes a new header

The new header assumes the right to collect the data from the member nodes and announces that it has become the header to them. Since every node in the network send data to the BS, and the BS broadcasts the information of the headers to all nodes while selecting the headers, it is better not to initiate the header selection algorithm too often. So the headset is maintained it is very likely that the node with the second-greatest remaining amount of energy becomes the header for the next round since each node of the cluster does not generally spend too much energy comparing with the 
amount of the initially given energy.

The proposed approach organizes clusters using the same mechanism of LEACH but different from LEACH where each of the headers permit to transmit data directly to BS, while new approach uses chaining to send data more efficiently as in PEGASIS. In the case of LEACH that the headers transmit data to the BS directly, the amount of energy spent for the transmission varies depending on the distance from the header to the BS.

Furthermore, the mechanism causes the headers to die early because the headers have to do both collecting the data from the members and sending them to the BS, and the number of transmission is more than other nodes do. In contrast, the proposed approach keeps the balance of the energy consumption among the headers since it chooses the leader node with the greatest amount of energy for data transmission, and consequently it helps the whole network last longer.

\section{Proposed Algorithm}

Step 1: Network Initialization

- Base Station broadcasts a low cost control messages for header selection to all nodes.

- All nodes send location and energy information to Base Station.

Step 2: Cluster Head - Set Selection

- BS selects a node with the greatest remaining energy becomes the first header for Cluster Head Set.

- Header Send the Advertisement

- Other Nodes reply to the header with Ack.

- Three Nodes with Maximum Energy are selected as a head set member.

Step 3: Path Chain Formation \& Leader Selection

- End Cluster active head sends the TOKEN to Next Cluster.

- Leader sends the TOKEN to Base Station

- Base Station broadcasts the 'chain completion' message.

Step 4: Data Transfer

- Member nodes of each cluster send data to Active Cluster Head.

- Active Cluster Heads collect the data.

- Active Cluster Heads send the collected data to the leader through the chain.

- Leader node sends the final gathered data to Base Station.

Step 5: Changing Active Header

- If $\mathrm{E}$ of Active Cluster Head $<\mathrm{E}_{\mathrm{th}}$, the Head set member with Maximum energy becomes a new header.

- If $\mathrm{E}$ of the three members is less than $\mathrm{E}_{\mathrm{th}}$, go to Step 2.

At Step 1, the BS sends a message to each node in the network, the member nodes reply with their location and remaining energy information, and the nodes transfer the requested data to the $\mathrm{BS}$. This process is the equivalent as the one in LEACH. Next, in the Step 2, the BS selects the headers based on the remaining energy information transferred in the Step 1. The selected headers spread the TDMA schedule to the neighbor nodes. However our new mechanism selects one header and the header send the advertisement to all other nodes, The nodes reply with the ACK, from that ACK the active head selects the Cluster Head set members, and hereafter when a new header needs to be selected, one of the head set member becomes a header without re-running the header selection algorithm.

The Step 3 is a process to form a chain along the headers decided in the previous steps. For this, we use the Greedy Algorithm used in PEGASIS. The chain is formed in the order from the furthest to the nearest node from the BS, and nearer nodes have better opportunities to be the leader. In the Step 4, through the chain of the headers formed in the Step 2 and 3, the collected data from the member nodes of each cluster are transferred by the their header, and the headers pass the data to their leader.

Continuously in the Step 5, when all members of head set have minimum energy than the threshold then a new header is selected using the header selection as in Step 2. However, since this protocol assumes that every node has the ability to directly transfer its data to the BS and that nodes have no mobility, it is realistically ineffectual. In Opposite to this, our new protocol can guarantee the mobility of the nodes as it periodically re-arrange the clusters over the whole network and the headers continuously report the state of their clusters to the BS.

\section{RESULTS AND DISCUSSION}

For evaluation, we used NS-2(ns-2.29.3) and tested proposed approach and other referred routing protocols, such as LEACH and PEGASIS. But, we assumed the following during the test.

1) The BS (Base Station) has powerful operation and data communication capabilities.

2) Every node has sensors and a transmitting device of the equivalent performance with each other.

3 ) The BS has the location information of all nodes.

4) Every node knows the distance to its neighbour nodes from itself.

5) Energy model is similar to PEGASIS.

6) Every node initially has $0.5 \mathrm{~J}$ of energy

7) For each transmission it requires50nJ per packer.

8) The maximum size of the packet is 2000 bits.

TABLE I: COMPARISION-ROUNDS IN WHICH FIRST NODE DIES

\begin{tabular}{llcrrr}
\hline No. of Nodes/ & \multicolumn{5}{l}{ Round in which the first node dies } \\
Algorithm & $\mathbf{5 0}$ & $\mathbf{1 0 0}$ & $\mathbf{1 5 0}$ & $\mathbf{2 0 0}$ \\
\hline Leach & 372 & 359 & 330 & 314 \\
Pegasis & 691 & 679 & 638 & 602 \\
Proposed Method & $\mathbf{7 3 8}$ & $\mathbf{7 1 7}$ & $\mathbf{6 6 1}$ & $\mathbf{6 2 8}$ \\
\hline
\end{tabular}

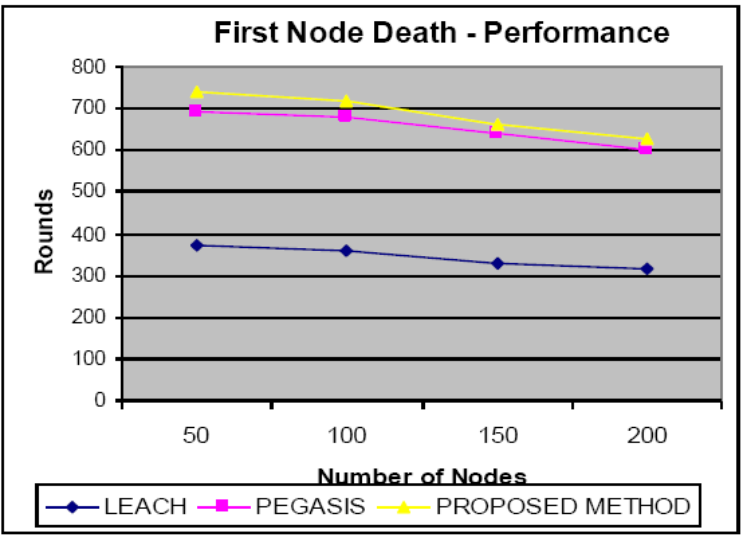

Fig. 2. Performance Test on Round at which the first node dies. 
In the Fig. 2, we displayed the result of the experiment that tested death of the first node in the network while the number of nodes was 50,100,150 and 200. The proposed approach works well with low density sensor network. And also it provides better result than LEACH and PEGASIS. For 100 Nodes the proposed algorithm gives $50 \%$ better performance than LEACH and $6 \%$ better performance than PAGASIS. In the Fig. 3, we displayed the result of the experiment that tested redundant transmission of the data. From the results we see that the proposed algorithm works well and reduces the redundant path, thereby minimize the energy usage and increasing the network life time.

\section{CONCLUSIONS}

In wireless sensor networks, the energy consumption and the network lifetime are important issues for the research of the route protocol. Among all protocols for sensor networks, LEACH and PEGASIS are known as the most energy-efficient algorithms. The disadvantage with LEACH is that headers spend a great amount of energy and they die earlier than other nodes. In PEGASIS if number of nodes is high and the chain become lengthy, the data transmission time from the source to the sink node, which is the BS, and the nodes close to the BS die earlier than others. In this paper, we tried solving the problems by combining PEGASIS and LEACH and introducing the concept of Cluster Head Set. More specifically, it divides nodes into clusters and selects the active headers and cluster head set that gather and transmit the data from their member nodes as in LEACH. After selecting the headers in this way, it forms a chain of the headers and sends data through the chain as in PEGASIS. Thus, with the new protocol suggested in this paper we could overcome the drawback of PEGASIS since the number of nodes forming the chain is smaller in new approach, and could minimize the overhead while clustering. Thus the new approach improves the network life time. For the future work, our research will focus on number of head set members and the method of transmission.

\section{REFERENCES}

[1] A. Chattopadhyay, M. Thomas, and A. Gupta, "An Energy Aware Routing Protocol for Mobile Ad-Hoc Networks," in Proc. 15th
International Conference on Advanced Computing and Communications, December 2007, pp. 262-267

[2] I. F. Akyildiz, W. Su, Y. Sankarasubramaniam, and E. Cayirci, "iA Survey on Sensor Networks," IEEE Communication Magazine, vol. 40, no. 8, August 2004, pp. 102-114.

[3] G. J. Pottle and W. J. Kaiseri, "Embedding the internet : wireless integrated network sensors," Communications of the ACM, vol. 43, no. 5, May 2000, pp. 51-58.

[4] A. A. Abbasia and M. Younisb, "A survey on clustering algorithms for wireless sensor networks," Computer Communications, vol. 30, no. 15, October 2007, pp. 2826-2841.

[5] W. R. Heinzelman, A. Chandrakasan, and H. Balakrishnan, "Energy-efficient communication protocol for wireless microsensor networks," in Proc. the Hawaii International Conference on System Science, Jan. 4, 2000.

[6] S. Lindsey and C. S. Raghavendra, "PEGASIS: power efficient gathering in sensor information systems," in Proc. IEEE Aerospace Conference, 2002, vol. 3, pp. 1125-1130.

[7] O. Younis and S. Fahmy, "HEED: a hybrid, energy-efficient, distributed clustering approach for ad hoc sensor networks," IEEE Trans. Mobile Computing, vol. 3, no. 4, pp. 366-379, Oct.-Dec. 2004

[8] S. Bandyopadhyay and E. J. Coyle, "An Energy Efficient Hierarchical Clustering Algorithm for Wireless Sensor Networks," in Proc. the IEEE INFOM2003, vol. 3. IEEE Press, New York, 2003, pp. 1713-1723.

[9] J.-H. Chang and L. Tassiulas, "Maximum lifetime routing in wireless sensor networks," IEEE/ACM Transactions on Networking, vol. 12, no. 4, Aug. 2004, pp. 609-619.

[10] J. Gong, H. Kim, and G. H. Cho, "A coverage efficient clustering method based on time delay for wireless sensor networks," in Proc. the 2008 International Conference on Wireless Networks, July 14-17, 2008, Las Vegas, US.

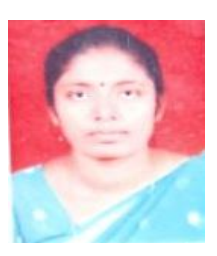

L. Malathi was born in India in 1982. She completed her bachelors of engineering degree in information technology during 2003 and the master of engineering degree in the Computer Science and Engineering Department in Anna University, Chennai during the year 2007. Her research interest includes wireless sensor networks, wireless Ad hoc network and QOS in multimedia.

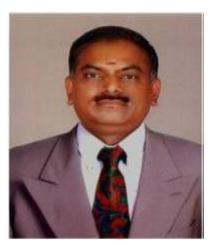

R. K. Gnanamurthy received his $\mathrm{PhD}$ degree in Faculty of Information and Communication Engineering from Anna University, Tamilnadu, India and M.E degree in microwave and optical engineering from Madurai Kamaraj University, Tamilnadu, India. Currently, he is a professor and principal at SKP Engineering College, Tiruvannamalai, Tamilnadu, India. He has published more than 90 papers including journals and conferences. His research areas include signal processing, pattern recognition, mobile networks, adhoc networks and VLSI circuits. 